\title{
A Silicon Differential Receiver With Zero-Biased Balanced Detection for Access Networks
}

\author{
Mariam Aamer, Nikolaos Sotiropoulos, Antoine Brimont, Jean-Marc Fedeli, Delphine Marris-Morini, \\ Eric Cassan, Laurent Vivien, Karen Ribaud, Philippe Grosse, Jean Michel Hartmann, \\ Diedrik Vermeulen, Gunther Roelkens, Pablo Sanchis, and Andreas Hakansson
}

\begin{abstract}
We present an optimized differential receiver in silicon with a minimized footprint and balanced zero-biased Ge photodiodes. The receiver integrates a delay-line with a $2 \times 4$ multimode interferometer $90^{\circ}$ hybrid and two balanced photodiodes for differential quadrature phase-shift keying demodulation. Two receivers are tested, for 10 and $20 \mathrm{~Gb} / \mathrm{s}$ operation, and well opened eye-diagrams and symbol constellations are obtained with error vector magnitude values as low as $12.5 \%$ and $19.57 \%$, respectively. The results confirm the potential of integrated silicon receivers to become key building blocks for future passive optical access networks based on advanced modulation formats.
\end{abstract}

Index Terms-Integrated optics, silicon photonics, balanced detectors, phase-shift keying.

\section{INTRODUCTION}

A $\mathrm{N}$ EVER growing amount of access network bandwidth is required by end users, and the deployment of passive optical networks, operating up to $10 \mathrm{Gbit} / \mathrm{s}$ has already begun to address this demand. Even higher bit rates will be required in the future, with network operators preferring solutions based on reusing the existing infrastructure and components developed for legacy links. Advanced modulation formats can enable the further scaling of the bit rates while keeping the legacy $10 \mathrm{GHz}$ devices [1].

Differential-phase-shift-keying (DPSK) offers several advantages over an on-off keying modulation format, namely

Manuscript received March 7, 2013; revised April 18, 2013 and May 6, 2013; accepted May 9, 2013. Date of publication May 16, 2013; date of current version June 13, 2013. This work was supported in part by the European Community's Seventh Framework Program under Grant 224312 HELIOS.

M. Aamer, A Brimont, and $\mathrm{P}$ Sanchis are with Nanotechnology Center Valencia, Universitat Politecnica, Valencia 46022, Spain (e-mail: maaa@ntc.upv.es; abrimont@ntc.upv.es; pabsanki@ntc.upv.es).

N. Sotiropoulos is with the Department of Electrical Engineering, Eindhoven University of Technology, Eindhoven 5600 MB, The Netherlands (e-mail: n.sotiropoulos@tue.nl).

J.-M. Fedeli, K. Ribaud, P. Grosse, and J. M Hartmann are with CEA, LETI, Grenoble 38054, France (e-mail: jean-marc.fedeli@cea.fr; karen.ribaud@cea.fr; philippe.grosse@cea.fr; jean-michel.hartmann@cea.fr).

D. Marris-Morinie, E. Cassan, and L. Vivien are with the Institut d'Electronique Fondamentale, Univ. Paris-Sud, CNRS, Orsay cedex F-91405, France (e-mail: delphine.morini@u-psud.fr; eric.cassan@u-psud.fr; laurent.vivien@u-psud.fr).

D. Vermeulen and G. Roelkens are with Photonics Research Group, INTEC, Ghent University/imec, Ghent 9000, Belgium (e-mail: Diedrik.Vermeulen@intec.ugent.be; gunther.roelkens@intec.ugent.be).

A. Hakansson is with DAS Photonics S.L, Ciudad Politecnica de la Inovacion, Valencia 46022, Spain (e-mail: ahakansson@dasphotonics.com).

Color versions of one or more of the fgures in this letter are available online at http://ieeexplore.ieee.org.

Digital Object Identifie 10.1109/LPT.2013.2262931 higher nonlinearity tolerance, $3-\mathrm{dB}$ receiver sensitivity improvement when used with balanced detection and the possibility to operate at lower symbol rates for a given bit rate using DQPSK [2]. The detection of the phase at the receiver can be implemented either by using coherent or differential (non-coherent) detection. The coherent receiver provides an improvement of the optical signal to noise ratio by mixing a local oscillator (LO) signal with the transmitted signal and effective compensation for signal degradations due to chromatic dispersion and polarization mode dispersion on the transmission line can be achieved. However, a digital signal processing (DSP) unit is necessary, which implies higher costs and electric power consumption. In the differential encoded DPSK, the information is encoded in the phase transition and can be demodulated using a passive delay-interferometer, which allows the elimination of the LO and the DSP, making differential detection advantageous for low-cost links.

Different approaches have been proposed for differential silicon receivers, mainly based on the use of standard MachZehnder delay interferometer (MZDI) [3]-[5] or using microring resonators [6], [7]. While the ring resonator approach allows a very compact implementation, optimal performance usually requires a tuning mechanism [8], increasing the complexity and power consumption of the receiver. Accordingly, the MZDI design seems to be the most suitable to be used in more complete systems [5], [9].

In this letter we demonstrate 5 GBaud $(10 \mathrm{Gbit} / \mathrm{s})$ and 10 GBaud (20 Gbit/s) operation using a very compact differential receiver for DQPSK demodulation with zero-biased balanced detection and using a compact $2 \times 4 \mathrm{MMI} 90^{\circ}$ hybrid instead of the standard implementation with two $180^{\circ}$ hybrids. $10 \mathrm{Gbit} / \mathrm{s}$ and $20 \mathrm{Gbit} / \mathrm{s}$ symbol constellations were obtained, as well as their error vector magnitude (EVM) and bit error rate (BER).

\section{Silicon DQPSK Receiver STRUCtURE}

The principle of operation of a differential demodulator using a MZDI is to interfere two adjacent symbols using a delay-line (DL) that introduces a time delay corresponding to one bit duration. This interference converts the phase difference between two consecutive symbols into an amplitude variation. In DQPSK modulation two bits are encoded as four different phase differences.

The schematic of the proposed receiver is depicted in Fig. 1. A thermo-optically tunable Mach-Zehnder interferom- 

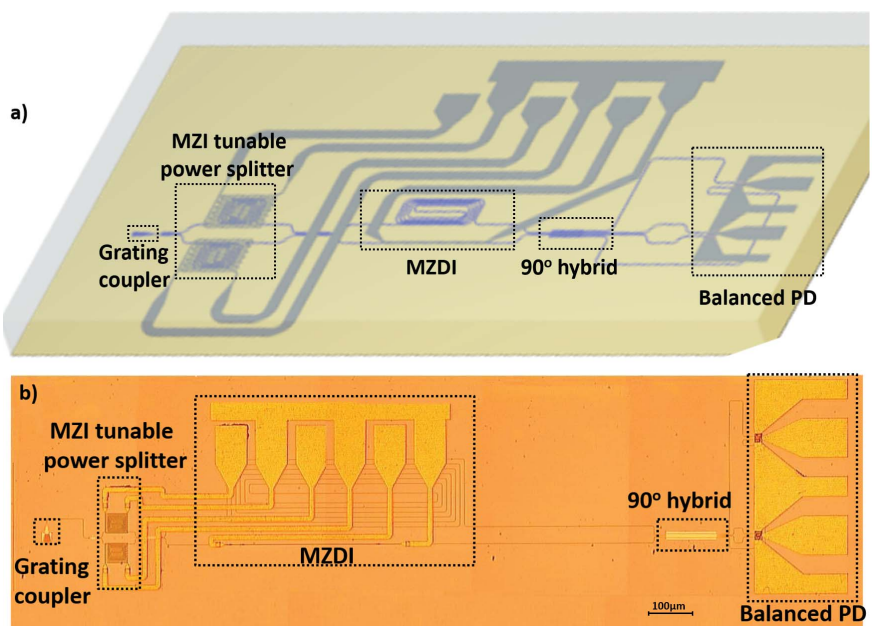

Fig. 1. DQPSK differential receiver. (a) Schematic and (b) optical photograph of the fabricated device. The receiver is assembled using 5 components, from left to right in the f gure: curved grating coupler, MZI tunable power splitter, delay line interferometer, $90^{\circ}$ hybrid and two balanced PDs.

eter (MZI) power splitter is placed in series with a MZDI and a $2 \times 4 \mathrm{MMI}$ acting as $90^{\circ}$ hybrid. At the output the in-phase and quadrature signals are detected using two balanced photodetectors (BPDs) in pinpin conf guration. In the following paragraphs the implementation and optimization of each of these elements is described.

The proposed device was fabricated on top of a $200-\mathrm{mm}$ SOI wafer with silicon core thickness of $220 \mathrm{~nm}$ and buried oxide of $2 \mu \mathrm{m}$. To couple light from the input $\mathrm{f}$ ber to the receiver, and in order to minimize the size of the chip, curved gratings [10] were used, showing a coupling loss of about $6 \mathrm{~dB}$. The second element of the receiver is a tunable MZI power splitter. In case of unbalanced behavior at the output of the MZDI due to excess loss in the delay line, micro-heaters can be used to actively tune the power at the MZDI input, resulting in an increase in the extinction ratio of the MZDI [11], and consequently in the sensitivity of the receiver. At the output of the power splitter, a MZDI was placed. Compact spirals were used in order to minimize the size of the structure. A waveguide length of $18 \mathrm{~mm}$ and $9 \mathrm{~mm}$ was chosen for $10 \mathrm{Gbit} / \mathrm{s}$ and $20 \mathrm{Gbit} / \mathrm{s}$ operation, respectively, introducing $\sim 3 \mathrm{~dB}$ and $\sim 1.5 \mathrm{~dB}$ propagation losses, respectively. The MZDI outputs were coupled to the $2 \times 4$ MMI inputs. This MMI, with an insertion loss of about $6.5 \mathrm{~dB}$, acted as a $90^{\circ}$ hybrid [12] instead of the standard implementation of a DQPSK receiver with two parallel MZDI coupled to a $180^{\circ}$ hybrid, in accordance with the intention of minimizing the chip area, as well as the number of active controls needed for tuning. In order to have a $3 \mathrm{~dB}$ increase in the sensitivity of the receiver and minimize power consumption, zero-bias balanced detection was used based on a Ge photodetector pair (Ge-BPD) in lateral pinpin conf guration. Hence, the photodetected signal is directly extracted from one single central pad avoiding additional wire bonding or metal connections. The $10 \mu \mathrm{m}$ length Ge-BPD was selectively grown at the end of silicon waveguides, and it is comparable to the ones used in [13]. The high-speed performance of the photodiodes used is comparable

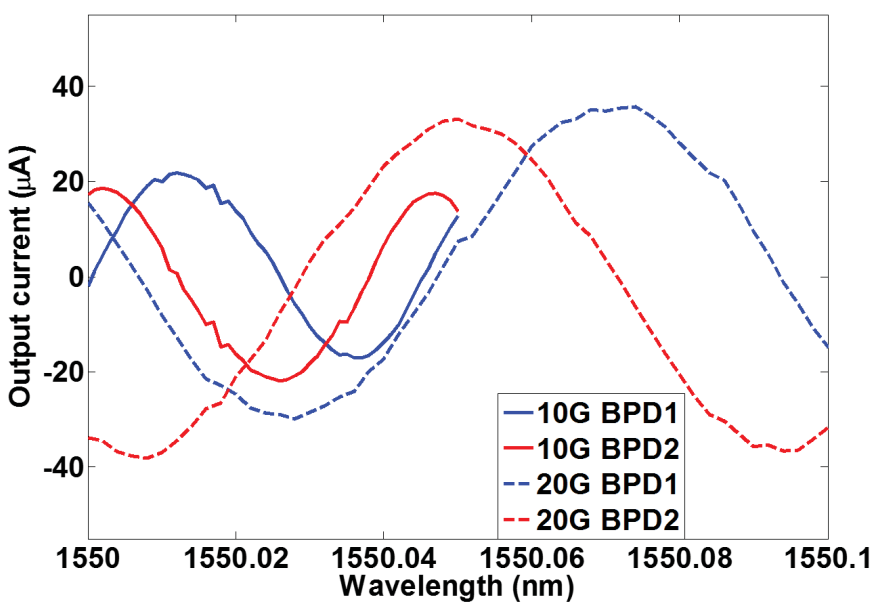

Fig. 2. Output current as a function of wavelength for the $10 \mathrm{Gbit} / \mathrm{s}$ (solid lines) and $20 \mathrm{Gbit} / \mathrm{s}$ (dashed lines) bit rate receiver. The blue and the red lines correspond to the in-phase (I) and quadrature (Q) outputs, respectively.

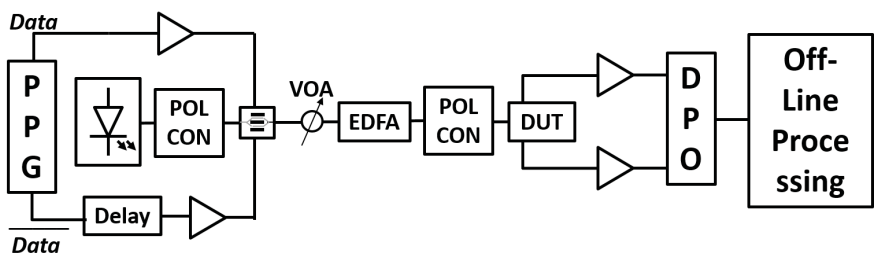

Fig. 3. Experimental setup for characterizing the DQPSK receiver. POL $\mathrm{CON}$ indicates polarization controller.

to the one having a bandwidth above $10 \mathrm{GHz}$ for any negative voltage bias, including $0 \mathrm{~V}$, which clearly is suff cient for our operation. The responsivity was measured to be $1 \mathrm{~A} / \mathrm{W}$ at $1550 \mathrm{~nm}$ using 0 to $-2 \mathrm{~V}$ bias. For an input power of $-3 \mathrm{dBm}$, the transmission spectra of the two BPDs were measured, for both receivers. As it can be seen in the fgure below, the spectra have excellent agreement with the expected behavior for a DQPSK receiver. For both receivers, the two outputs are perfectly $90^{\circ}$ phase shifted with respect to each other conf rming the correct behavior of the $90^{\circ}$ hybrid.

The performance, in terms of responsivity, of our Ge-BPDs is quite better than those presented in [3], [4], [6]. On the other hand, the total optical excess loss of our proposed device is around $15 \mathrm{~dB}$ and $16.5 \mathrm{~dB}$ for the 10 and $20 \mathrm{Gbit} / \mathrm{s}$, respectively, values of the same order as those presented in [5].

\section{EXPERIMENTAL RESULTS}

The setup used to test the high-speed behavior of the receiver is shown in Fig. 3. A DQPSK signal was generated using a commercial single-drive Lithium Niobate nested MZ modulator, biased at minimum transmission and driven by the outputs of the pulse pattern generator (PPG), appropriately decorrelated, aligned and amplif ed at $2 \mathrm{~V}_{\pi}$. The bits were generated from a pseudorandom binary sequence pattern generator (PRBS) with a pattern length of $2^{13}-1$, which was the longest sequence that could be used to create a custom pattern sequence. This custom sequence was used to allow for the insertion of a preamble that facilitated the discovery of the start of the sequence of the detected data. The generated DQPSK 

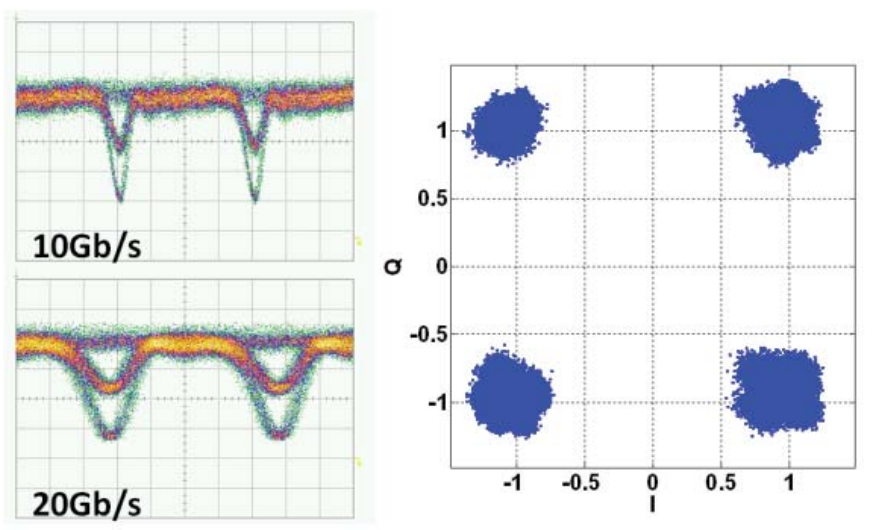

Fig. 4. $10 \mathrm{Gbit} / \mathrm{s}$ and $20 \mathrm{Gbit} / \mathrm{s}$ transmitted DQPSK eyes, and reference constellation measured by a discrete receiver $(20 \mathrm{Gbit} / \mathrm{s})$.
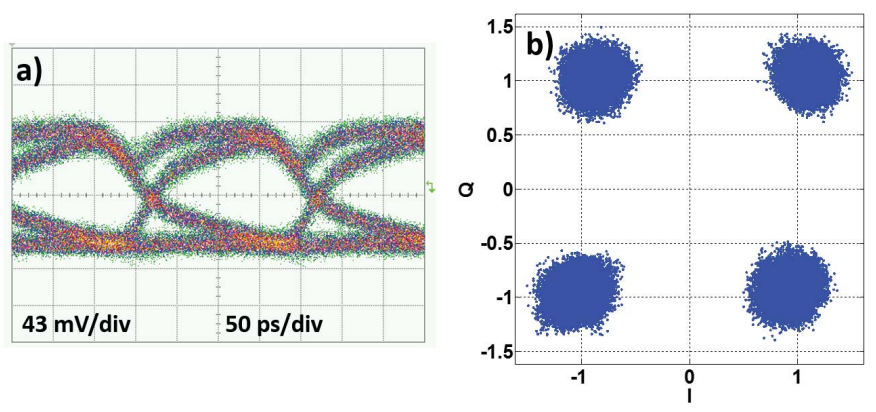

Fig. 5. (a) DQPSK eye diagram and (b) symbol constellationat $10 \mathrm{Gbit} / \mathrm{s}$ for a received power of $-19 \mathrm{dBm}$.

signal passes through a variable optical attenuator (VOA), which is the reference point for the received power, and is then amplif ed by an erbium-doped f ber amplif er (EDFA). At the output of the chip, the electrical signals were extracted directly from the photodiodes using RF probes in a GSGSG conf guration, and were transferred to two amplif ers, and then were sampled by the digital phosphor oscilloscope (DPO) for further processing off-line.

The DQPSK transmitted eyes at 10 and $20 \mathrm{Gbit} / \mathrm{s}$ are shown in Fig. 4, as well as the reference constellation obtained by using a discrete receiver described in [14].

For the $10 \mathrm{Gbit} / \mathrm{s}$ integrated receiver, well opened eyediagrams and remarkably good constellation diagrams were obtained, as shown in Fig. 5 for a received power of $-19 \mathrm{dBm}$.

It must be commented that in long haul networks, the parameter of interest lies in the relation between the BER and the optical signal to noise ratio, due to the fact that the signal is usually degraded by noise introduced by a large number of amplifier . However, in an access network, the figur of merit is the sensitivity as a function of received power, as there is no degradation due to amplificati $\mathrm{n}$ stages and the receiver sensitivity limits the possible splitting ratio and reach of the system. The EVM as a function of the received power was measured and the experimental results are summarized in Table I.

As the quality of the received signal was quite good so that no signif cant amount of errors were recorded in the captured length of the data, which was $100 \mathrm{k}$ symbols, the measured
TABLE I

EVM AND BER, FOR THE 10 GBIT/S DifFERENTIAL RECEIVER

\begin{tabular}{|c|c|c|}
\hline Received Power $(\mathrm{dBm})$ & Measured EVM (\%) & Estimated BER \\
\hline-23 & 24.27 & $1.9 \times 10^{-5}$ \\
\hline-22 & 22.39 & $4 \times 10^{-6}$ \\
\hline-21 & 14.76 & $6.2 \times 10^{-12}$ \\
\hline-20 & 13.08 & $10^{-14}$ \\
\hline $\mathbf{- 1 9}$ & $\mathbf{1 2 . 5}$ & $\mathbf{1 0}^{-15}$ \\
\hline
\end{tabular}
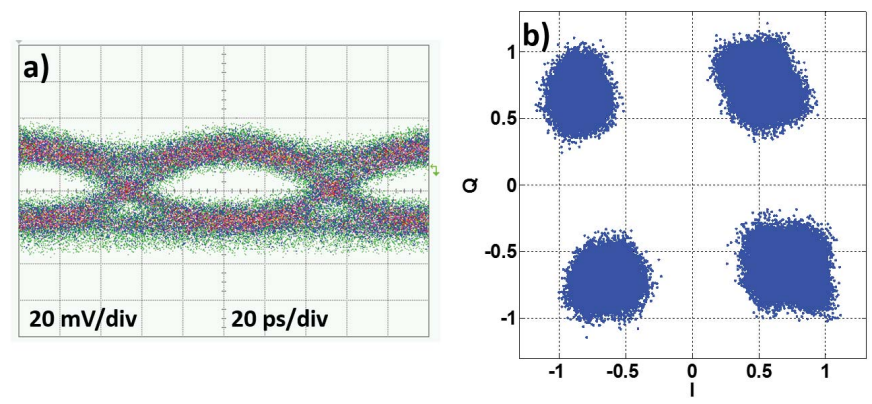

Fig. 6. (a) DQPSK eye diagram and (b) symbol constellation at $20 \mathrm{Gbit} / \mathrm{s}$ for a received power of $-12 \mathrm{dBm}$.

TABLE II

EVM AND BER, FOR THE 20 GBIT/S DifFERENTIAL RECEIVER

\begin{tabular}{|c|c|c|}
\hline Received Power $(\mathrm{dBm})$ & Measured EVM (\%) & Estimated BER \\
\hline-14 & 25.66 & $6 \times 10^{-5}$ \\
\hline-13 & 19.68 & $1.9 \times 10^{-7}$ \\
\hline $\mathbf{- 1 2}$ & $\mathbf{1 9 . 5 7}$ & $\mathbf{1 . 7} \times \mathbf{1 0}^{-7}$ \\
\hline
\end{tabular}

EVM of the signal was used to estimate the BER by using the following equation taken from [15]:

$$
B E R \approx \frac{\left(1-\frac{1}{L}\right)}{\log _{2} L} \operatorname{erfc}\left[\sqrt{\left.\left[\frac{3 \log _{2} L}{L^{2}-1}\right] \frac{1}{E V M^{2} \log _{2} M}\right]}\right.
$$

where $L$ is the number of signal levels per quadrature, $M$ is the order of the modulation and $Q$ indicates the $Q$ function. For QPSK modulation, $L$ is 2 and $M$ is 4 .

It must be commented that for input powers lower than $-21 \mathrm{dBm}$, the EDFA in Fig. 3 can no longer amplify the signal to a constant output power value of $+16 \mathrm{dBm}$, and lower powers reach the balanced photodiodes. Even so, and examining the results shown in the table above, an error foor value of around $12.5 \%$ and $10^{-15}$ were obtained for the EVM and BER, respectively.

For the $20 \mathrm{Gbit} / \mathrm{s}$ DQPSK receiver, opened eyes and good constellation diagram were also measured, as shown in Fig. 6 for a received power of $-12 \mathrm{dBm}$. Comparing the obtained constellation diagram with the reference one shown in Fig. 4, it can be seen that some additional noise appears in the symbols of the constellations. Nevertheless, the EVM of the obtained constellation was measured to be $19.57 \%$ and the corresponding BER was estimated by using Eq. (1) in $1.7 \cdot 10^{-7}$, as shown in Table II. It must be taken into account that typically, advanced modulation formats operate with Forward-Error Correction (FEC), and our BER values, without FEC, are below the $10^{-3}$ reference, which is the 
FEC limit for 10G-Ethernet Passive Optical Network (EPON) standard [16], [17].

\section{CONCLUSION}

A minimum footprint differential receiver for DQPSK transmission with zero-biased detection for access networks has been demonstrated at 10 and $20 \mathrm{Gbit} / \mathrm{s}$. Each building block of the receiver was optimized for high performance and maximum compactness. The results indicate the potential of integrated silicon receivers to become key building blocks for future passive optical access networks based on advanced modulation formats. Furthermore, the integration of the receiver with transimpedance amplifier would improve its sensitivity and so higher-order modulation formats could be supported due to the low EVM values achieved. For instance, for an EVM of $12.5 \%$, translated into BER for D8PSK, results into a BER lower than $10^{-5}$ [15].

\section{ACKNOWLEDGMENT}

The authors would like to thank P. Crozat from IEF, and Dr. J. Herrera and R. Sambaraju for fruitful discussion.

\section{REFERENCES}

[1] F. Effenberger, "The XG-PON system: Cost effective $10 \mathrm{~Gb} / \mathrm{s}$ access," J. Lightw. Technol., vol. 29, no. 4, pp. 403-409, Feb. 15, 2011.

[2] A. H. Gnauck and P. J. Winzer, "Optical phase-shift-keyed transmission," J. Lightw. Technol., vol. 23, no. 1, pp. 115-130, Jan. 2005.

[3] M. Kroh, et al., "Integrated receivers on silicon-on-insulator for advanced modulation formats," IET Optoelectron., vol. 5, no. 5, pp. 211-217, Oct. 2011.

[4] S. Faralli, K. N. Nguyen, J. D. Peters, D. T. Spencer, D. J. Blumenthal, and J. E. Bowers, "Integrated hybrid Si/InGaAs $50 \mathrm{~Gb} / \mathrm{s}$ DQPSK receiver," Opt. Express, vol. 20, no. 18, pp. 19726-19734, 2012.
[5] C. R. Doerr, N. K. Fontaine, and L. L. Buhl, "PDM-DQPSK silicon receiver with integrated monitor and minimum number of controls," IEEE Photon. Technol. Lett., vol. 24, no. 8, pp. 697-699, Apr. 15, 2012.

[6] K. Xu, Z. Cheng, C. Y. Wong, and H. K. Tsang, "Tunable integrated variable bit-rate DPSK silicon receiver," Opt. Lett., vol. 37, no. 22, pp. 4738-4740, Nov. 2012.

[7] Y. Ding, et al., "Multi-channel $40 \mathrm{Gbit} / \mathrm{s}$ NRZ-DPSK demodulation using a single silicon microring resonator," J. Lightw. Technol., vol. 29, no. 5, pp. 677-684, Mar. 1, 2011.

[8] L. Chen, N. Sherwood-droz, and M. Lipson, "Compact bandwidthtunable microring resonators," Opt. Lett., vol. 32, no. 22, pp. 3361-3363, Nov. 2007.

[9] K. Suzuki, H. C. Nguyen, T. Tamanuki, F. Shinobu, Y. Sakai, and T. Baba, "Slow-light-based variable symbol-rate silicon photonics DQPSK receiver," Opt. Express, vol. 20, no. 4, pp. 4796-4804, Feb. 2012.

[10] F. Van Laere, et al., "Compact focusing grating couplers for siliconon-insulator integrated circuits," IEEE Photon. Technol. Lett., vol. 19, no. 23, pp. 1919-1921, Dec. 1, 2007.

[11] M. Aamer, A. Griol, A. Brimont, A. Gutierrez, P. Sanchis, and A. Håkansson, "Increased sensitivity through maximizing the extinction ratio of SOI delay-interferometer receiver for 10G DPSK," Opt. Express, vol. 20, no. 13, pp. 14698-14704, Jun. 2012.

[12] R. Halir, G. Roelkens, A. Ortega-Moñux, and J. G. WangüemertPérez, "High-performance $90^{\circ}$ hybrid based on a silicon-on-insulator multimode interference coupler," Opt. Lett., vol. 36, no. 2, pp. 178-180, Jan. 2011.

[13] L. Vivien, et al., "Zero-bias 40Gbit/s germanium waveguide photodetector on silicon," Opt. Express, vol. 20, no. 2, pp. 1096-1101, Jan. 2012.

[14] N. Sotiropoulos, T. Koonen, and H. de Waardt, "TDM-PON with $30 \mathrm{~Gb} / \mathrm{s}$ D8PSK downstream and $10 \mathrm{~Gb} / \mathrm{s}$ OOK upstream based on a digital incoherent receiver," Opt. Express, vol. 20, no. 27, pp. 29096-29104, Dec. 2012.

[15] R. Schmogrow, et al., "Corrections to "Error vector magnitude as a performance measure for advanced modulation formats," IEEE Photon. Technol. Lett., vol. 24, no. 23, p. 2198, Dec. 1, 2012.

[16] 10-Gigabit-Capable Passive Optical networks (XG-PON): Transmission Convergence (TC) Layer Specificatio , Standard ITU-T G.987.3.

[17] E. I. De Betou, et al., "Upstream FEC performance in combination with burst mode receivers for next generation $10 \mathrm{Gbit} / \mathrm{s}$ PON," in Proc. ECOC, 2010, Sep., pp. 1-3. 